\title{
Multicriteria Decision Making for Enhanced Perception-Based Multimedia Communication
}

\author{
Gheorghita Ghinea, Member, IEEE, George D. Magoulas, Member, IEEE, and Christos Siamitros
}

\begin{abstract}
This paper proposes an approach that integrates technical concerns with user perceptual considerations for intelligent decision making in the construction of tailor-made multimedia communication protocols. Thus, the proposed approach, based on multicriteria decision making (MDM), incorporates not only classical networking considerations, but, indeed, user preferences as well. Furthermore, in keeping with the task-dependent nature consistently identified in multimedia scenarios, the suggested communication protocols also take into account the type of multimedia application that they are transporting. Lastly, this approach also opens the possibility for such protocols to dynamically adapt based on a changing operating environment and user's preferences.
\end{abstract}

Index Terms-Application-aware adaptation, multimedia video, quality of perception, uncertainty.

\section{INTRODUCTION}

M ATCHING user-level requirements with parameters characterizing underlying network performance is a problem rarely studied in multimedia communications, for it attempts to bridge the gap existing between user perceptions of multimedia quality, on the one hand, and the quality of service (QoS) with which multimedia is transmitted over the network, on the other.

The concept of QoS in distributed multimedia systems is indelibly associated with the provision of an acceptable level of application performance. In turn, this performance is itself dependent on both the perceived subjective quality of the application as well as its robustness to network congestion.

The focus of this research has been the enhancement of the traditional view of QoS with a user-level-defined quality of perception (QoP). This is a measure that encompasses not only a user's satisfaction with multimedia clips, but also his/her ability to perceive, synthesize, and analyze the informational content of such presentations. As such, the interaction between QoP and QoS and its implications from both a user perspective [1] as well as a networking angle [2] have been investigated. Although the problem of multimedia application-level performance is

Manuscript received December 18, 2003; revised June 7, 2004. The work of G. D. Magoulas was supported in part by the Engineering and Physical Sciences Research Council (EPSRC), U.K. This paper was recommended by Associate Editor Guerlain

G. Ghinea is with the Department of Information Systems and Computing, Brunel University, Uxbridge, Middlesex UB8 3PH, U.K. (e-mail: george. ghinea@brunel.ac.uk).

G. D. Magoulas was with Brunel University, Uxbridge, Middlesex UB8 3PH, U.K. He is now with the School of Computer Science and Information Systems, Birkbeck College, University of London, London WC1E 7HX, U.K. (e-mail: g.magoulas@dcs.bbk.ac.uk).

C. Siamitros is with the Department of Mathematics, Brunel University, Uxbridge, Middlesex UB8 3PH, U.K. (e-mail: christos.siamitros@brunel. ac.uk).

Digital Object Identifier 10.1109/TSMCA.2005.851281 closely linked to both the user perspective of the experience as well as to the service provided by the underlying network, it is rarely studied from an integrated viewpoint. Clearly, this is a very unsatisfactory state of affairs.

In this paper, this situation is tried and rectified by proposing a scheme for QoP management based on an integrated architecture that takes into account both user-centric QoP and low-level QoS parameters. Such a scheme would then, by appropriate management of these QoS parameters, provide the potential of ensuring an optimum QoP in a distributed multimedia setting.

In the next section, the reasons behind the current segregated approach and some attempts that have been made to redress this imbalance shall be investigated more closely. Section III summarizes empirical results in the measurement of human QoP given a multimedia presentation. Section IV describes the framework that shall be used as a test bed for the new approach and shows how multicriteria decision making (MDM) can be used to obtain a QoP-oriented ordering of QoS parameters, while Section $V$ provides application examples of this approach. Lastly, conclusions are drawn in Section VI.

\section{BACKGROUND AND MOTIVATION}

The networking foundation on which current distributed multimedia applications are built either do not specify QoS parameters (also known as best effort service) or specify them in terms of traffic engineering parameters such as delay, jitter, and loss or error rates. However, these parameters do not convey application-specific needs such as the influence of clip content and informational load on the user multimedia experience. As a result, the underlying network does not consider the sensitivity of the application performance to bandwidth allocation. There is thus an architectural gap between the provision of networklevel QoS and application-level QoP requirements of the distributed multimedia applications. This gap causes distributed multimedia systems to inefficiently use network resources and results in poor end-to-end performance that, in turn, has a direct negative impact on the user experience of multimedia.

Previous work in the area of subjective quality assessment of multimedia applications has concentrated on the entertainment side of multimedia. Accordingly, work has been done to appraise the enjoyment of multimedia presentations shown at differing frame rates [3], while [4] examined the effect that random media losses have on user-perceived quality. The bounds within which lip synchronization can fluctuate without undue annoyance on the viewer's part have also been studied [5], as has the establishment of metrics for subjective assessment of teleconferencing applications [6]. 
The inherent difficulty of trying to link subjective sentiments about the quality of the presentation with the facts and figures of network parameters is reflected in the relative scarcity of work trying to bridge the application-network gap. In essence, three approaches can be identified. The first approach tries to bridge the application-network gap implicitly. By this, it is understood that there is no explicit mapping between application-level user requirements and the QoS provided by the network. What happens, instead, is that the user specifies, usually through a graphical user interface, his/her desired presentation quality [7], [8]. This is typically provided through the means of sliders or radio buttons via which the user would specify, for example, the desired playback frame rate, spatial resolution, as well as the acceptable synchronization delay between the audio and video streams.

In the second approach, an explicit mapping, linking application-level user requirements to network QoS, is actually given. Such a mapping can either be defined on a per layer basis, such as a network to transport to session to applicationlayer mapping, or directly between application and networklevel parameters [9].

The last approach is, in essence, a more restrictive version of the first. What happens here is that the user is played shortduration probes of differing deliverable qualities of the multimedia material in question and (s)he then specifies which of the given sample qualities is acceptable [9]. Apart from the obvious goal of polling user-preferred multimedia quality, the probebased approach is advantageous from the point of view that it tests current network conditions. Thus, any choice that the user might make in as far as desired multimedia quality goes is guaranteed to be delivered - at least in the initial stages-by the network.

In this paper, the problem of bridging the applicationnetwork gap from a multiattribute decision-making perspective is addressed. The present approach was used to integrate results from the authors' work on user-level QoP with the more technical characterization of QoS. The ultimate aim is to provide a communications architecture that uses an adaptable communications protocol geared towards human requirements in the delivery of distributed multimedia.

\section{USER-PERCEIVEd QuAlity OF SERVice}

This approach of evaluating user-perceived QoS (QoP) has been mainly empirical, as is dictated by the fact that its primary focus is on the human side of multimedia computing. What has been done is that users from diverse backgrounds (test subjects involved academics, students, secretaries, businessmen) and ages (12-58 years old) were presented with a set of four short (30-45-s duration) multimedia clips, snapshots of which are shown in Fig. 1. These were chosen to be varied, ranging from a relatively static weather forecast clip to a highly dynamic action movie sequence. All of them depicted excerpts from realworld programs and thus represent informational sources that an average user might encounter in everyday life. Each clip was shown with the same set of QoS parameters, unknown to the user. After each clip, the user was asked a series of questions (ranging from 10 to 12 ) based on what had just been seen, and
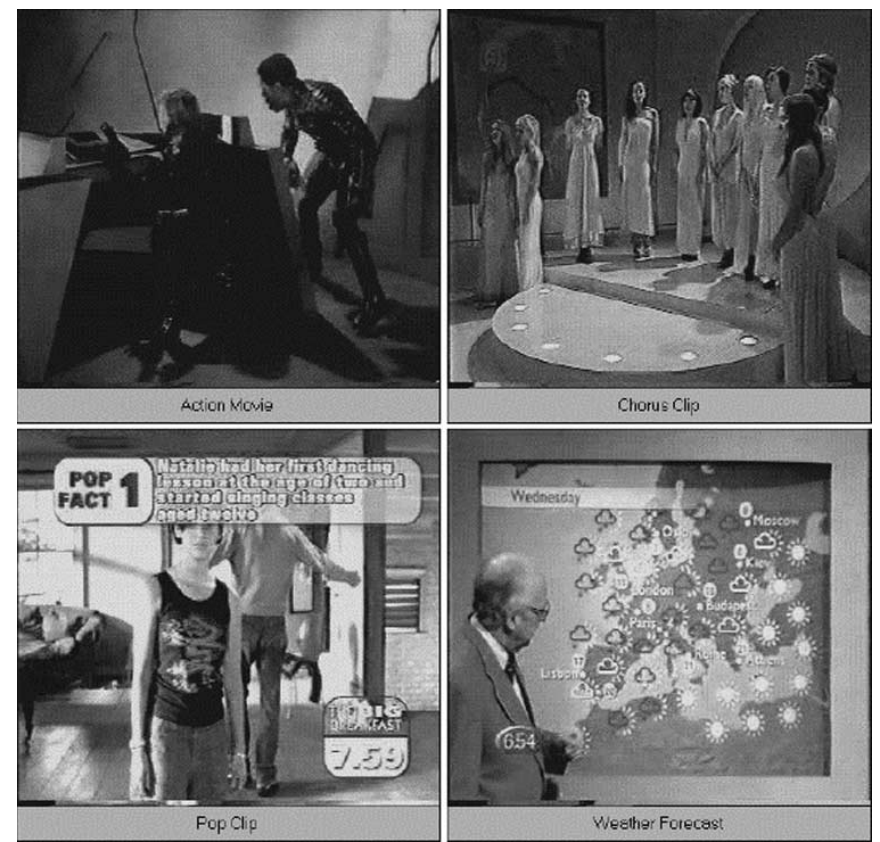

Fig. 1. Snapshots of multimedia clips used in the experiments.

the experimenter duly noted the answers. Lastly, the user was asked to rate the quality of the clip that had just been seen on a scale of 1-6 (with scores of 1 and 6 representing the worst and best perceived qualities possible, respectively).

Because of the relative importance of the audio stream in a multimedia presentation as well as the fact that it takes up an extremely low amount of bandwidth compared to the video, it was decided to transmit audio at full quality during the experiments. Parameters were, however, varied in the case of the video stream. These include both spatial parameters (such as color depth) and temporal parameters (frame rate). Accordingly, two different color depths were considered ( 8 and 24 bits), together with three different frame rates $[5,15$, and 25 frames per second (fps)]. Twelve users have been tested for each (frame_rate, color_depth) pair. In summary, the results (see [1] for a more detailed coverage) obtained in the QoP experiments show that:

1) A significant loss of frames (that is, reducing the frame rate) does not proportionally reduce the user's understanding and perception of the presentation. In fact, in some instances, (s)he seemed to assimilate more information, thereby resulting in more correct answers to questions. This is because the user has more time to view a frame before the frame changes (at $25 \mathrm{fps}$, a frame is visible for only $0.04 \mathrm{~s}$, whereas at $5 \mathrm{fps}$, a frame is visible for $0.2 \mathrm{~s}$ ), hence absorbing more information. This observation has implications on resource allocation.

2) User assimilation of the informational content of clips is characterized by the wyswyg (what you see is not what you get) relation. What this means is that, often, users, while still absorbing information correctly, do not notice obvious cues in the clip, a phenomenon known as "inattentional blindness" [10]. Instead, the reasoning process by which they arrive at their conclusions is based a lot on intuition and past experience. 
3) Users have difficulty in absorbing audio, visual, and textual information concurrently. Moreover, if the user perceives problems with the presentation (such as lip synchronization) users will disregard them and focus on the audio message if that is considered to be contextually important. This implies that critical and important messages in a multimedia presentation should be delivered in only one type of medium, or, if delivered concurrently, should be done so with maximal possible quality.

4) Highly dynamic scenes, although expensive in resources, have a negative impact on user understanding and information assimilation. Questions in this category obtained the least number of correct answers. However, the entertainment value of such presentations seems to be consistent, irrespective of the frame rate at which they are shown. The link between entertainment and content understanding is therefore not direct, and this is further confirmed by the second observation above.

All these results indicate that technical-oriented QoS must also be specified in terms of perception, understanding, and absorption of content-QoP in short-if multimedia presentations are to be truly effective.

Therefore, in linking perceptual considerations with lowlevel technical parameters, the design process should take into account the subjective judgement of the end-user. However, the end-user may not clearly specify a desired parameter value and may prefer the use of linguistic phrases to either describe their priorities, e.g., "Audio is equally important as Text," "Video is slightly more important than Audio," or to answer more vague questions, such as "How important is guaranteed audio for this clip?" Moreover, in the case of a technically aware user, more complicated questions like "How much more preferable is a flow control protocol when compared to a checksum algorithm with respect to obtaining a satisfactory audio quality?" and "How important is a specific acknowledgement scheme for user perception of video?" can also be asked. This approach does result in inherent imprecision, though. Although information about questions like the previous ones is vital in making correct design decisions, it is very difficult, if not impossible, to quantify them correctly, i.e., the main problem is how to quantify the linguistic choices made by the user. In the next section, a method that, from combined user-, application- and networklevel requirements, ultimately results in a communication protocol configuration, specifically tailored for the respective user needs, will be described.

\section{User-Centered Design With MUlTiCRITERIA CONSTRAINTS}

Distributed guaranteed services need to incorporate capabilities for responding to QoP and QoS changes originating from the user/applications or the system/network. To achieve these changes, the networked multimedia system will require fast renegotiation protocols and adaptive mechanisms. The renegotiation protocols will rely on dependable and simple monitoring and recognition algorithms to detect requests for QoS changes or system degradations. The envisioned adaptive mechanisms should include update mechanisms for resource
TABLE I

ADAPTABLE FUnCTIONALITY IN DRoPS

\begin{tabular}{cc}
\hline \hline Protocol Mechanism & Implementations \\
\hline Sequence control & none $\mid$ complete \\
Flow control & none | window based \\
Acknowledgement scheme & IRQ | PM-ARQ \\
Checksums & none | block checking | full CRC \\
\hline \hline
\end{tabular}

allocation in response to detection of system degradation [11]. To this end, a framework that allows for not only runtime construction of tailored multimedia communication protocols, but also, through the incorporation of MDM, for the inclusion of user requirements in such protocols, is presented.

\section{A. Framework for QoP Adaptation}

The dynamically reconfigurable stacks project (DRoPS) provides an infrastructure for the implementation and operation of multiple adaptable protocols [12]. The core architecture is embedded within the Linux operating system, is accessible through standard interfaces, such as sockets and the UNIX ioctl (I/O control) system calls, has direct access to network devices and benefits from a protected multiprogramming environment. The architecture allows additional QoS maintenance techniques, such as flow shaping (to smooth out bursts in traffic), at the user or interface level, and transmission queue scheduling, at the device queue level.

DRoPS-based communication protocols are composed of fundamental mechanisms, called microprotocols, which perform arbitrary protocol processing operations. The complexity of processing performed by a microprotocol is not defined by DRoPS and may range from a simple protocol function, such as a checksum, to a complex layer of a protocol stack, such as TCP. In addition, protocol mechanisms encapsulated within a microprotocol may be implemented in hardware or software. If appropriate hardware is available, the microprotocol merely acts as a wrapper, calling the relevant hardware function. Microprotocols are encapsulated in loadable modules, allowing code to be dynamically loaded into a running operating system and executed without the need to recompile a new kernel. Each such microprotocol can be implemented via a number of adaptable functions, as detailed in Table I. In particular, microprotocols may also represent the absence of a particular function, such as the one representing no sequence control, as shown in Table I.

While a protocol defines the structure and resources available for constructing a communication system, a protocol stack defines a unique instantiation assigned to a particular connection. In terms of microprotocols, a protocol stack is an ordered set combined to form a functional communication system. Each connection is assigned a protocol stack for its sole use, the configuration of which may vary according to the characteristics of the particular connection. Using this model, individual flows within individual sessions may be uniquely configured to provide an appropriate service. Thus, a connection between video client and server applications may use a semantically strong protocol for commands and a relatively weak one for bulk transfer of relatively loss tolerant graphical data. The 
overhead of adaptation has been measured at $2.8 \mu \mathrm{s}$, is justified by the overall improvement in performance that adaptation yields, and is well within real-time constraints.

The DRoPS framework does not place restrictions on the implementation of particular protocol functionalities. For instance, an acknowledgement protocol can be implemented either as an idle repeat request (IRQ) or a per message acknowledgement scheme (PM-ARQ). However, the decision behind implementation choices of particular protocols is not straightforward, for it has to deal with inherent imprecision either at the network or user levels. A mechanism is needed to handle such situations, and the analytic hierarchy process (AHP), described in Section IV-B, is particularly suited in this respect.

\section{B. Analytic Hierarchy Process Formalism}

Intelligent decision making in the construction of communication protocols is achieved by adopting the AHP formalism, which is one of the most popular methods of the MDM.

The AHP formalism, originally proposed in [14], has been successfully applied in solving real-world decision-making problems involving generation of a set of alternatives, setting priorities, choosing the best policy after finding a set of alternatives, allocating resources, determining requirements, predicting outcomes, designing systems, and resolving conflicts. The capability to handle subjective criteria and inconsistencies in the decision-making process and the conceptual simplicity of that method are the major reasons of its popularity.

The present approach based on the AHP formalism may be better appreciated through the following quotation [13]: "The theory [of the AHP] reflects what appears to be an innate method of operation of the human mind. When presented with a multitude of elements, controllable or not, which comprise a complex situation, it aggregates them into groups, according to whether they share certain properties. [...] These elements may, in turn, be grouped according to another set of properties, generating the elements of yet another 'higher,' level, until we reach a single 'top' element which can often be identified as the goal of out decision-making process."

What was just described in this Saaty's quotation is commonly called a hierarchy, i.e., a set of elements or factors arranged in levels. A key question now arises: What is the amount of influence of each factor belonging to the lowest level of the hierarchy on the top factor or overall goal? Answering this question leads to identifying various levels of intensity, as influence is not uniformly distributed over the various factors, which are usually called priorities.

From a more technical point of view, the AHP is implemented through three steps [13], [15], [17]. The first step is structuring of the hierarchy, which as mentioned earlier corresponds to determining the relative factors, i.e., criteria and alternatives (see Fig. 2). To this end, the first level of the hierarchy is used to denote the overall objectives or goals of the decision problem. The second level is occupied by criteria for assessing the accomplishment of the objectives, while the third level contains available actions or alternatives.

The second step in AHP is pairing of comparisons to yield preference weights or priorities. The main task of this stage is to determine numerical measures to the relative importance of the criteria and to the relative performance of the alternatives on these criteria. It consists of the following two subprocedures.

1) Determine the relative importance of the criteria.

2) Determine the relative standing of each alternative with respect to each criterion.

The final stage of the AHP is synthesis of preference weights to yield composite priorities for alternatives.

In the following, the use of this formalism within the DRoPS framework is described.

\section{Multicriteria Decision Making for QoP Managements}

According to the experimental QoP results discussed in Section III, multimedia QoP varies with the number of media flows, the type of medium, the type of application, and the relative importance of each medium in the context of the application. Thus, within the QoP framework, each multimedia application can be characterized by the relative importance of the video $(V)$, audio $(A)$, and textual $(T)$ components as conveyors of information, as well as the dynamism $(D)$ of the presentation. On the other hand, consistent with the DRoPS framework, five network level QoS parameters have been considered in this model: bit-error-rate (BER), segment loss (SL), segment order (SO), delay (DEL) and jitter (JIT). Together with the $V, A, T$, and $D$ parameters, these constitute, in Saaty's terminology, the criteria on the basis of which an appropriate tailored communication protocol is constructed. In DRoPS, as shown in Table I, the functionality of this protocol is realized through a number of nine microprotocols, spanning four broad functionality classes [2].

By applying Saaty's methodology, a total of ten matrices is obtained. Nine of these matrices give the relative importance of the various microprotocols (alternatives, in Saaty's vocabulary) with respect to the criteria identified in the present model, while the last of these matrices details pairwise comparisons between the criteria themselves.

Psychological experiments have shown that individuals cannot simultaneously store in their working memory more than seven objects $( \pm 2)$ [18]. Thus, usually, pairwise comparisons are quantified by using a scale of nine grades, which describe the relative importance of the criteria [13]. If $a_{i j}$ is an element on this nine-point scale, i.e., $a_{i j} \in\{1,2,3, \ldots, 8,9\}$, then $a_{i j}=1 / a_{j i}$ also holds [13]

$$
A^{\mathrm{BER}}=\left[\begin{array}{lllllllll}
1 & 1 & 1 & 1 & 1 & 1 & 1 & \frac{1}{3} & \frac{1}{9} \\
1 & 1 & 1 & 1 & 1 & 1 & 1 & \frac{1}{3} & \frac{1}{9} \\
1 & 1 & 1 & 1 & 1 & 1 & 1 & \frac{1}{3} & \frac{1}{9} \\
1 & 1 & 1 & 1 & 1 & 1 & 1 & \frac{1}{3} & \frac{1}{9} \\
1 & 1 & 1 & 1 & 1 & 1 & 1 & \frac{1}{3} & \frac{1}{9} \\
1 & 1 & 1 & 1 & 1 & 1 & 1 & \frac{1}{3} & \frac{1}{9} \\
1 & 1 & 1 & 1 & 1 & 1 & 1 & \frac{1}{3} & \frac{1}{9} \\
3 & 3 & 3 & 3 & 3 & 3 & 3 & 1 & \frac{1}{5} \\
9 & 9 & 9 & 9 & 9 & 9 & 9 & 5 & 1
\end{array}\right] .
$$

An example of one of the former types of matrices, i.e., of the different alternatives with respect to one of the criteria 


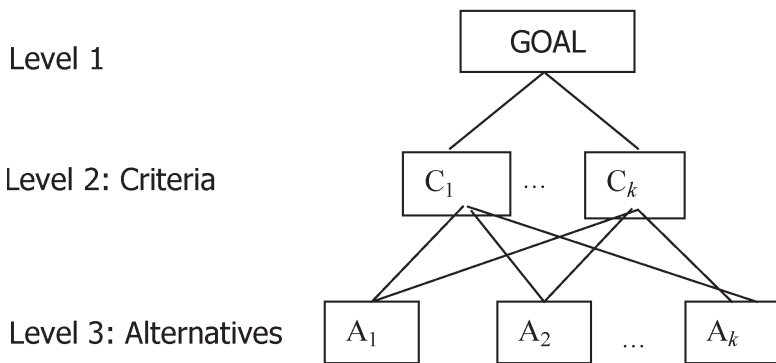

Fig. 2. Analytic hierarchy process.

(bit error rate in this case) is given in (1). Each entry $a_{i j}$ of the matrix $A^{\mathrm{BER}}$ represents a designer-defined numerical judgement giving the relative suitability of various alternatives to fulfil a specified criterion.

For example, in (1), the judgement "microprotocol 1 is equally important as microprotocol 2 with respect to BER" corresponds to a weighting of $a_{i j}=1$, while the judgement "microprotocol 9 is absolutely more important than microprotocol 1" would correspond to a value of $a_{i j}=9$. Intermediate terms can also be assigned when compromise is needed between two adjacent characterizations. Note that in (1), the considered microprotocols are, in order: \{no sequence control, strong sequence control, no flow control, window-based flow control, IRQ, PM-ARQ, no checksum algorithm, block checking, full cyclic redundancy check (CRC) \}. For example, as far as bit error rate is concerned [see (1)], the only microprotocols that have an impact upon it are the checksum algorithms. The strongest of these methods, the full CRC, has the highest weight (a value of $a_{i j}=9$ ) in comparison with all the others, while a relatively weak block checking algorithm is considered to be moderately more important $\left(a_{8 j}=3, j=1,2, \ldots, 7\right)$ than microprotocols from other functionality classes.

While all the previous nine matrices considered have a constant form, the matrix of each criterion with respect to all the other criteria named $C$, shown below, is the only one whose values may fluctuate as a result of changes in the operating environment, as well as a consequence of changes in user preferences and perceptions. Relation (2) provides an instance of this matrix used in the present model; the respective criteria are, in order, BER, SO, SL, DEL, JIT, $V, A, T$, and $D$

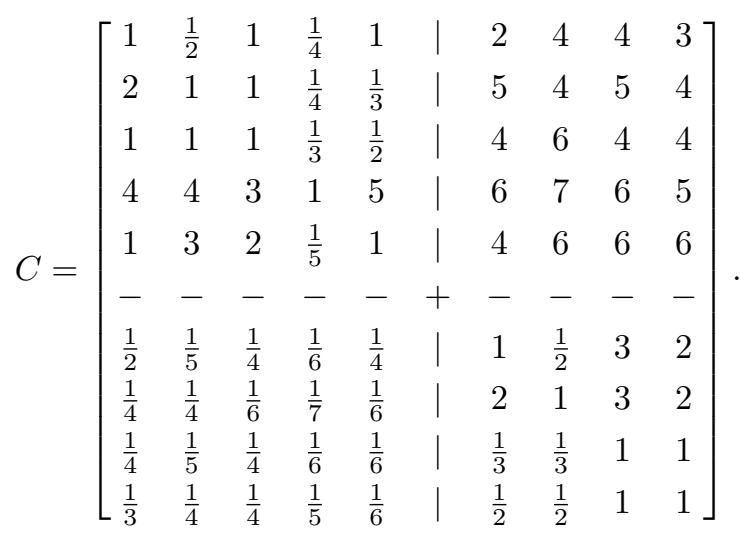

An average user, though, would have difficulty in a priori judgement of varying technical parameters such as delay, jitter, error, and loss rates on highly subjective attributes such as perception, understanding, and satisfaction. While this is true for QoS attributes at the level of the transport service, users are better able to quantify their requirements in terms of more abstract characteristics like the prioritization of core multimedia components such as $V, A$, and $T$. The matrix in (2) reflects this situation and could conceptually be split into four submatrices, which are:

1) A $5 \times 5$ matrix, in the upper left part of matrix $C$ [see (2)], giving the relative importance of the BER, SO, SL, DEL, and JIT criteria with respect to one another. This matrix changes dynamically during the course of the transmission of a multimedia clip. For example, an intelligent controller may, as a result perhaps of a delayintolerant audio application being subjected to a period of high network delays, change the numerical judgements of the submatrix to reflect a more radical bias in favor of the delay component.

2) A $4 \times 4$ matrix, in the lower right part of the matrix given by (2). Here, user input can reflect personal choices of the relative importance of the video, audio, and textual components in the context of the application, as well as a relative characterization of the dynamism of the multimedia clip. While these values can be changed dynamically depending on the visualized scene, a priori values in this case could reflect the result of user consultations. This indeed is the case with the present QoP experiment [1], where a broad base of people were polled about their opinions for the range of clips presented in Fig. 1. Thus, users characterized the action movie clip as being highly dynamic, i.e., dynamism is high, with a similar importance being attached to the video component, while the audio was considered to convey a medium informational load, in contrast to the text component that was judged to have a low informational weight.

3) A $5 \times 4$ and a $4 \times 5$ matrix that reflect the designer choices of the relative importance of the five QoS parameters considered on $V, A, T$, and $D$. The elements of these matrices reflect the results of a previous work on QoP and define the balance between the relative importance of QoP and QoS parameters [1].

Following the AHP, the priority weights $w_{i}, i=1, \ldots, 9$ denoting the relative importance of each criterion $i$ among the $p=9$ criteria (a higher priority setting corresponds to a greater importance) can be evaluated using different weight determination procedures, such as the eigenvalue method [19], the logarithmic least squares method [19], the goal programming method [20], or the fuzzy programming methods (FPMs) [21].

Pairs among alternatives are also compared with respect to the $i$ th criterion and then a weight $w_{j, i}$, which denotes how preferable the alternative $j$ is with respect to the criterion $i$, is derived. As discussed previously, there is a total of $p(p-1) / 2$ pairwise comparisons in the matrix and weights can be calculated using any one of the methods [19]-[21]. At this point, it is important to note that the quality of the weighted priorities is highly affected by the consistency of the judgements of the decision maker. When QoP and QoS judgements are perfectly 
consistent, then all the elements $a_{i j}$ have perfect values, and the consistent priorities are unique.

However, in the present case, the evaluations $a_{i j}$ are frequently not perfect, as they are just estimations based on the best available data. Furthermore, as a result of the dynamic nature of the present problem, there are cases when the technical information and the perceptual information introduce inconsistencies in the judgment matrices. Thus, a weight determination technique suitable to handle inconsistencies is indispensable, as will be explained in Section IV-D.

Finally, the weighted sum model [19] is used to find the preference of an alternative $j$ with respect to all criteria simultaneously; preference is defined by $P_{j}$ and denotes the overall priority, or weight, of action $j$

$$
P_{j}=\sum_{i=1}^{p} w_{i} \cdot w_{j, i}
$$

Obviously, in the maximization case, the best alternative is the one that possesses the highest priority value among all others.

\section{Multicriteria Decision Making and the Fuzzy Programming Method}

The dynamic nature of the present problem requires the use of a weight determination technique able to handle inconsistencies. Therefore, the FPM, which is a method capable to solve even highly inconsistent matrices, was used [21], [22].

FPM is based on a geometrical representation of the prioritization process as an intersection of hyperlines and determines the values of the priorities, corresponding to the common intersection point of all hyperlines. In case of inconsistent matrices, the hyperlines have no common intersection point; i.e., the intersection set is empty. Thus, FPM represents the hyperlines as fuzzy lines and finds the solution of the approximate priority assessment problem, as an intersection point of these fuzzy lines, i.e., it finds a fuzzy intersection region that contains many points with different degrees of membership in this region and determines the values of the priorities, corresponding to the point with the highest measure of intersection. In [22], it is shown that FPM is able to produce better results than other methods when the degree of inconsistency is high.

Usage of the FPM enables judgements to be expressed either as crisp, intervals, or fuzzy numbers. Each reciprocal pairwise comparison matrix $A=\left[a_{i j}\right] \in \Re^{p \times p}$ can be represented as a system of $m=p(p-1)$ linear equalities

$$
R w=0
$$

where $n$ is the number of elements compared, $w$ is the vector of priority weights, and $R \in \Re^{m \times p}$. For the inconsistent cases, the FPM finds a solution that approximately satisfies (4), i.e., $R w \approx 0$.

One of the most important advantages of the FPM is that the prioritization problem is reduced to a fuzzy programming problem that can be easily formulated and solved as a standard linear programming problem

$$
\begin{aligned}
& \text { Obj. : } \max \lambda \\
& \text { s.t. } \lambda d_{k}+\mathrm{R}_{k} w \leq d_{k}, \quad k=1, \ldots, m, \quad 1 \geq \lambda \geq 0 \\
& \quad \sum_{i=1}^{p} w_{i}=1, \quad w_{i}>0, \quad i=1, \ldots, p
\end{aligned}
$$

where $\lambda$ is the degree of satisfaction of the constraints $R$ and the values of the tolerance parameters $d_{k}$ represent the admissible interval of approximate satisfaction of the crisp inequalities $\mathrm{R}_{k} w<0$. For the practical implementation of the FPM, it is reasonable for all these parameters $d_{k}$ to be set equal [22]. The optimal solution to (5) is a vector $\left(w^{*}, \lambda^{*}\right)$, whose first component maximizes the degree of membership of the fuzzy feasible area set and whose second component gives the value of the maximum degree of satisfaction. The method is explained in details in [21].

After deriving the underlying weights from the comparison matrices through the FPM technique, the priority weights $w_{i}$ and the relative scores $w_{j, i}$ are synthesized following the weight-sum model [19]. The overall priority value $P_{j}$ of the $j$ th alternative $\mathrm{A}_{j}(j=1, \ldots, k)$ is expressed as in (3). Obviously, the alternative with the maximum overall value $P_{j}$ will be chosen.

\section{ARchitecture for QoP MANAgEment}

For the construction of adaptable tailor-made protocols, we have integrated the DRoPS framework with AHP formalism into an architecture that combines QoS and QoP considerations and is able to intelligently manage user QoP, bearing in mind the dynamically fluctuating QoS. The diagram of this architecture is given in Fig. 3 and shows how both monitored QoS and user choices impact on the construction of the judgement matrix, which serves as the basis for the AHP to suggest a suitable protocol stack configuration under DroPS, ensuring that user QoP is maintained at an optimum level [23], [24]. This is in contrast to traditional legacy protocols stack such as TCP/IP and UDP, which make no allowance for user-related considerations in their functionality.

In its existing form, the present architecture uses the FPM described above to solve a nine-criteria and nine-alternative communication protocol construction problem. In the present approach, the end-user interacts in the evaluation of the criteria judgement matrix. In particular, the judgement matrix consists of three parts: one that deals exclusively with QoP issues; one that deals solely with QoS judgements; and one that reflects the balance between QoP and QoS considerations (Fig. 3). As mentioned above, within the QoP framework, each multimedia application can be characterized by the relative importance of the $V, A, T$, and its $D$. At this point, it should be mentioned that the QoP part of the judgement matrix is the only part evaluated by the end-user according to his preference regarding his/her priorities attached to the four components considered 


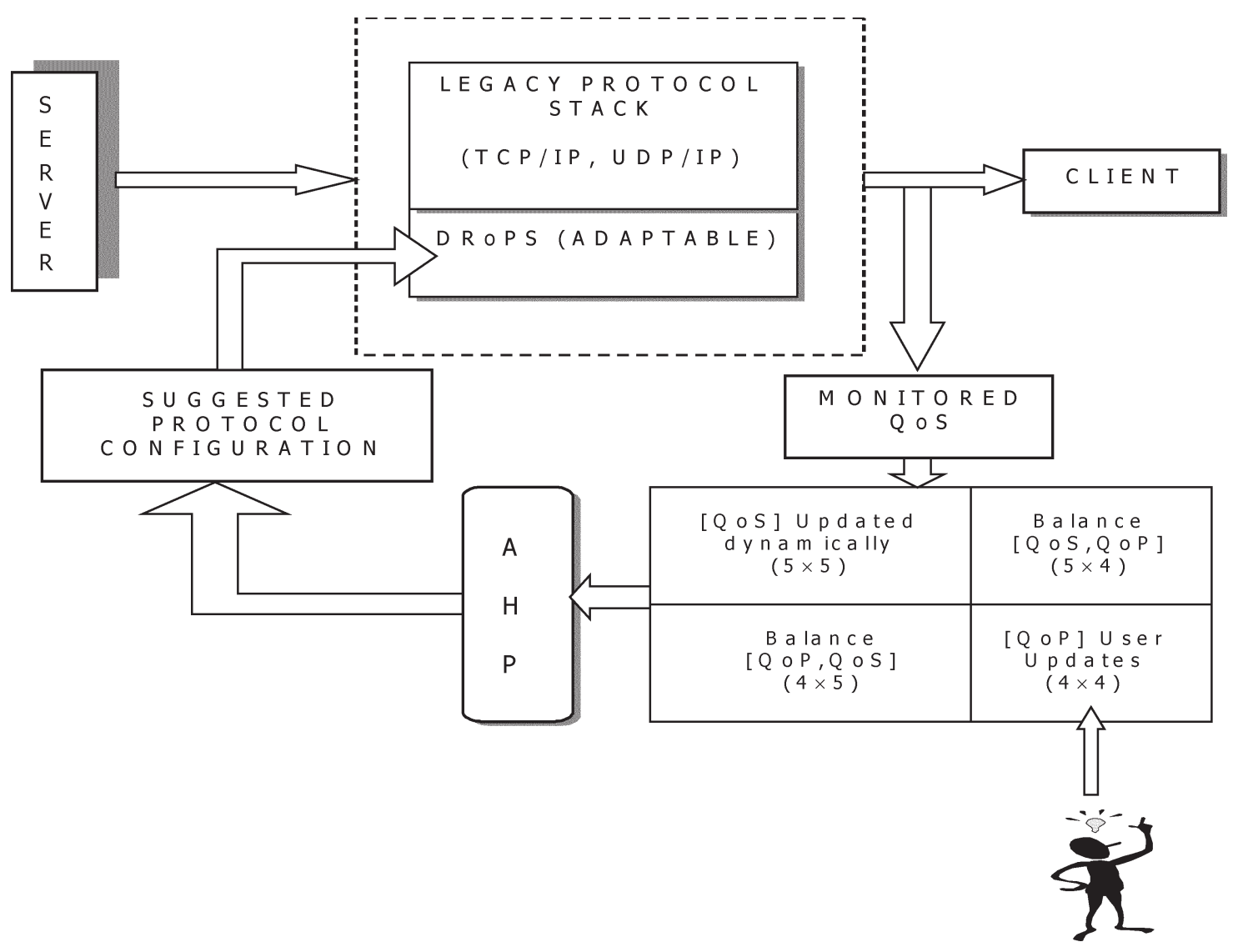

Fig. 3. AHP-based architecture for QoP management.

in the present model. In the QoS part, five network level QoS parameters are considered: BER, SL, SO, DEL, and JIT.

\section{EXPERIMENTS AND RESULTS}

In this section, experiments illustrating the ability of the present approach to select appropriate microprotocols and construct a suitably tailored protocol stack depending on the prevailing operating network environment are presented.

In the experiments, the following nine microprotocols, as given in Table I, namely no sequence control (micro1), strong sequence control (micro2), no flow control (micro3), window-based flow control (micro4), IRQ (micro5), PM-ARQ (micro6), no checksum algorithm (micro7), block checking (micro8), and full CRC (micro9) were pairwise compared with respect to one criterion each time to create the nine alternative judgement matrices. The pairwise relations are coded using the following conventions: $1 \rightarrow$ "equally important" (EQI); $2 \rightarrow$ "slightly more important" (SMI); 1/2 $\rightarrow$ "slightly less important" (SLI); $3 \rightarrow$ "weakly more important" (WMI); $1 / 3 \rightarrow$ "weakly less important" (WLI); $4 \rightarrow$ "moderately more important" (MMI); 1/4 $\rightarrow$ "moderately less important" (MLI); $5 \rightarrow$ "strongly more important" (SMI); $1 / 5 \rightarrow$ "strongly less important"; $6 \rightarrow$ "essentially important" (EI); 1/6 $\rightarrow$ "essentially less important" (ELI); 7 $\rightarrow$ “demonstrably important" (DI); $1 / 7 \rightarrow$ "demonstrably less important" (DLI); $8 \rightarrow$ "highly important" (HI); 1/8 $\rightarrow$ "highly less important" (HLI); $9 \rightarrow$ "absolutely important" (AI); 1/9 $\rightarrow$ "absolutely less important" (ALI).
For instance, in Table II, the microprotocols are compared with respect to audio $(A)$. As it can be observed from this table, microprotocols 1, 3, and 7 are of the same importance and are also the most important protocols with respect to the audio criterion. All the nine alternative matrices were evaluated and solved by applying the FPM (5).

The relative scores $w_{j, i}$ thus obtained are presented in Table III, where, for example, one can notice that the first four microprotocols considered have an equal importance with respect to managing SL. However, the most important microprotocol for segment loss is microprotocol 6 , which has the highest relative score. The formulation of all of the ten matrices that have been used in the present approach has been based on the results of the experiments with users described in Section III, and involved 15 users replying to questionnaires in order to elicit their judgements and subjective priorities. Here, users had to directly specify media priorities [resulting in the lower right submatrix of (2)], while the technical judgements resulted out of discussions with a group of four experts. This process is undertaken only once, with users subsequently being able to dynamically change, within the DRoPS adaptation constraints and according to their preferences, only the lower right submatrix of (2).

The applicability of the present approach with four scenarios is illustrated, depicting different networking conditions and user requirements. In the first scenario, a general case where the network adapts based on previously established judgement values is considered, and the judgement matrix shown in Fig. 3 
TABLE II

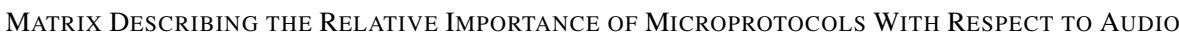

\begin{tabular}{lccccccccc}
\hline \hline Audio & micro1 & micro2 & micro3 & micro4 & micro5 & micro6 & micro7 & micro8 & micro9 \\
\hline micro1 & EQI & EI & EQI & EI & WMI & EI & EQI & WMI & EI \\
micro2 & ELI & EQI & MLI & EQI & EQI & EQI & DLI & EI & WMI \\
micro3 & EQI & MMI & EQI & EI & WMI & EI & EQI & WMI & EI \\
micro4 & ELI & EQI & ELI & EQI & WMI & ELI & DLI & ELI & WLI \\
micro5 & WLI & EQI & WLI & WLI & EQI & WMI & WLI & EQI & WMI \\
micro6 & ELI & EQI & ELI & EI & WLI & EQI & ELI & WMI & EQI \\
micro7 & EQI & DI & EQI & DI & WMI & EI & EQI & MMI & EI \\
micro8 & WLI & ELI & WLI & EI & EQI & WLI & MLI & EQI & EI \\
micro9 & ELI & WLI & ELI & WMI & WLI & EQI & ELI & ELI & EQI \\
\hline \hline
\end{tabular}

TABLE III

Relative Scores $w_{j, i}$ OF the Alternative Comparison Matrices With Respect to Each Criterion

\begin{tabular}{lccccccccc}
\hline \hline & \multicolumn{10}{c}{ Relative Scores } \\
& micro1 & micro2 & micro3 & micro4 & micro5 & micro6 & micro7 & micro8 & micro9 \\
\hline BER & 0.0574 & 0.0574 & 0.0574 & 0.0574 & 0.0574 & 0.0574 & 0.0574 & 0.1541 & 0.4441 \\
SO & 0.1082 & 0.2754 & 0.0787 & 0.0787 & 0.1049 & 0.1180 & 0.0787 & 0.0787 & 0.0787 \\
SL & 0.0881 & 0.0881 & 0.0881 & 0.0881 & 0.1872 & 0.2753 & 0.0617 & 0.0617 & 0.0617 \\
DEL & 0.2280 & 0.0638 & 0.2280 & 0.0638 & 0.0517 & 0.0456 & 0.2280 & 0.0456 & 0.0456 \\
JIT & 0.1614 & 0.1958 & 0.0771 & 0.3335 & 0.0474 & 0.0316 & 0.0743 & 0.0474 & 0.0316 \\
$V$ & 0.0842 & 0.2254 & 0.0842 & 0.1883 & 0.0979 & 0.0818 & 0.0462 & 0.0694 & 0.1226 \\
$A$ & 0.2175 & 0.0658 & 0.2175 & 0.0658 & 0.0590 & 0.0568 & 0.2529 & 0.0354 & 0.0293 \\
$T$ & 0.1761 & 0.0833 & 0.1761 & 0.0833 & 0.1250 & 0.0833 & 0.1761 & 0.0625 & 0.0341 \\
$D$ & 0.0843 & 0.0826 & 0.0826 & 0.3710 & 0.0759 & 0.0759 & 0.0759 & 0.0759 & 0.0759 \\
\hline \hline
\end{tabular}

TABLE IV

JudgemEnt MATRIX DESCRIBING THE RELATIVE IMPORTANCE OF QOS AND QOP PARAMETERS

\begin{tabular}{llllllllll}
\hline \hline & BER & SO & SL & DEL & JIT & $V$ & $A$ & $T$ & $D$ \\
\hline BER & EQI & SLI & SLI & DLI & ELI & EQI & EQI & EQI & EQI \\
SO & SMI & EQI & EQI & ELI & SLI & EQI & EQI & EQI & EQI \\
SL & SMI & EQI & EQI & ELI & SLI & EQI & EQI & EQI & EQI \\
DEL & DI & EI & EI & EQI & SMI & EQI & EQI & EQI & EQI \\
JIT & EI & SMI & SMI & SLI & EQI & EQI & EQI & EQI & EQI \\
$V$ & EQI & EQI & EQI & EQI & EQI & EQI & ELI & WLI & SLI \\
$A$ & EQI & EQI & EQI & EQI & EQI & EI & EQI & SMI & SMI \\
$T$ & EQI & EQI & EQI & EQI & EQI & WMI & SLI & EQI & EQI \\
$D$ & EQI & EQI & EQI & EQI & EQI & SMI & SLI & EQI & EQI \\
\hline \hline
\end{tabular}

does not suffer modifications. The second scenario depicts a case showing how users with special needs can use the present approach to create suitably tailored multimedia communication protocol stacks for their perceptual needs. The third scenario shows how the present method caters for extreme network conditions, while the last scenario illustrates how this approach handles dynamically changing multimedia content for the perceptual benefit of the user.

\section{A. Scenario 1: General Case}

In this example, no assumptions are made about the underlying network conditions, or about the multimedia content to be transported over the network. As such, the a priori judgement values that arise from technical considerations, as
TABLE V

Priority Weights $w_{i}$ With Respect to the Criteria $(i=1, \ldots, 9)$

\begin{tabular}{ccccccccc}
\hline \hline \multicolumn{8}{c}{ Priority Weights } \\
BER & SO & SL & DEL & JIT & $V$ & $A$ & $T$ & $D$ \\
\hline 0.0858 & 0.0951 & 0.0951 & 0.1573 & 0.1105 & 0.0936 & 0.1495 & 0.1066 & 0.1066 \\
\hline \hline
\end{tabular}

well as QoP judgements resulting out of the present QoP evaluation experiments [1], are used and are given in Table IV. As it can be observed from the table, delay and audio are the most important criteria from a technical and user point of view, respectively. This is because distributed multimedia applications have an essential real-time characteristic, which makes delay the primordial factor from the technical point of view. On the other hand, this work on perceptual aspects of multimedia has confirmed previous experiments in highlighting that the most important medium in a multimedia presentation, from a user's perspective, is the audio component.

Furthermore, from a technical angle, the criterion of SL has the same importance as the criterion of the SO. This is reflected in Table IV, where these two criteria are shown to be EQI. Similarly, for the user, the criterion of text $(T)$ is as important as dynamism $(D)$ ( $T$ and $D$ are EQI). Moreover, as can be observed, QoS and QoP parameters are considered to be EQI. This fact reflects a balance between QoP and QoS considerations in this initial scenario.

By applying the FPM (5), the priority weights $w_{i}$ are derived (see Table V). Finally, by synthesizing the relative scores 
TABLE VI

RANKING ORDER OF THE NINE MICROPROTOCOLS IN TERMS of the OVERALl Priority $P_{j}(j=1, \ldots, 9)$

\begin{tabular}{cc}
\hline \hline Microprotocol & Overall Priority \\
\hline micro1 & 0.1454 \\
micro4 & 0.1435 \\
micro3 & 0.1331 \\
micro7 & 0.1313 \\
micro2 & 0.1198 \\
micro9 & 0.0897 \\
micro6 & 0.0861 \\
micro5 & 0.0855 \\
micro8 & 0.0655 \\
\hline \hline
\end{tabular}

TABLE VII

JudgEMENT MATRIX DESCRIBING THE RELATIVE IMPORTANCE OF QOS AND QOP PARAMETERS FOR A VISUALLY IMPAIRED USER OF MULTIMEDIA

\begin{tabular}{llllllllll}
\hline \hline & BER & SO & SL & DEL & JIT & $V$ & $A$ & $T$ & $D$ \\
\hline BER & EQI & SLI & SLI & DLI & ELI & EQI & DLI & EQI & EQI \\
SO & SMI & EQI & EQI & ELI & SLI & EQI & DLI & EQI & EQI \\
SL & SMI & EQI & EQI & ELI & SLI & EQI & DLI & EQI & EQI \\
DEL & DI & EI & EI & EQI & SMI & EQI & DLI & EQI & EQI \\
JIT & EI & SMI & SMI & SLI & EQI & EQI & DLI & EQI & EQI \\
$V$ & EQI & EQI & EQI & EQI & EQI & EQI & DLI & WLI & SLI \\
$A$ & DI & DI & DI & DI & DI & DI & EQI & DI & DI \\
$T$ & EQI & EQI & EQI & EQI & EQI & WMI & DLI & EQI & EQI \\
$D$ & EQI & EQI & EQI & EQI & EQI & SMI & DLI & EQI & EQI \\
\hline \hline
\end{tabular}

(see Table III) and the priority vector, using (3), the overall priorities (overall scores) of the alternatives are obtained (see Table VI).

Microprotocol 1 is suggested as the best alternative, as it has a high relative score for both delay (the most important parameter from a technical/QoS point of view) as well as audio (the most important parameter from a user/QoP point of view). Although, as can be observed, microprotocol 7 is even better suited to manage these two parameters, if one considers the overall set of parameters, it can be observed that microprotocol 1 achieves at least equal or higher relative scores than microprotocol 7 for eight out of the nine parameters/criteria (see Table III).

\section{B. Scenario 2: Universal Access}

In the second scenario, it is assumed that a user with special accessibility requirements would need to play the multimedia clips considered in this work. It would thus seem logical for a visually impaired user to specify that the video and textual components of the multimedia presentation are not of paramount importance, whereas audio becomes "demonstrably important." An analogous situation would occur, for instance, if a hearingimpaired user would try to play the clips-here, (s)he might specify that the video stream is of primordial importance. In the former scenario, it is expected that the present approach outputs as its preferred outcome a microprotocol capable of handling the respective criterion identified as being of demonstrable importance relative to the other user-centered criteria (audio, text, clip dynamism) used in the present model.

Thus, in the case of audio being identified as demonstrably important, the audio-related entries of the criteria with respect
TABLE VIII

Priority Weights $w_{i}$ With Respect to the Criteria $(i=1, \ldots, 9)$ WHEN AUdio IS “DEMONSTRABLY IMPORTANT”

\begin{tabular}{ccccccccc}
\hline \hline & \multicolumn{10}{c}{ Priority Weights } \\
BER & SO & SL & DEL & JIT & $V$ & $A$ & $T$ & $D$ \\
\hline 0.0426 & 0.0481 & 0.0481 & 0.0862 & 0.0585 & 0.0554 & 0.5142 & 0.0735 & 0.0735 \\
\hline \hline
\end{tabular}

TABLE IX

RANKING ORDER OF THE MICROPROTOCOLS AND CORRESPONDING Overall PrioRity $P_{j}(j=1, \ldots, 9)$ When Audio IS "DEMONSTRABLY IMPORTANT"

\begin{tabular}{cc}
\hline \hline Microprotocol & Overall Priority \\
\hline micro7 & 0.1843 \\
micro1 & 0.1766 \\
micro3 & 0.1701 \\
micro4 & 0.1131 \\
micro2 & 0.0954 \\
micro5 & 0.0742 \\
micro6 & 0.0726 \\
micro9 & 0.0614 \\
micro8 & 0.0522 \\
\hline \hline
\end{tabular}

to the criteria matrix reflect this user requirement by taking values in the set $\{$ DI, DLI $\}$ as shown in Table VII.

The fact that user intervention has resulted in a new criteria with respect to the criteria matrix has no impact on the evaluation of the relative scores, $w_{j, i}$ (see Table III), which depends on the nine constant alternatives with respect to each criterion judgment matrix. However, user intervention affects the evaluation of the priority weights $w_{i}$. The new priority weights vector that resulted after applying the FPM (5) is shown in Table VIII. Finally, the overall priority values when audio is "demonstrably important" are calculated by applying (3), and they are shown in Table IX. As one can see, the most important microprotocols (alternatives) with respect to audio are micro7, micro1, and micro3 (in this order) with overall priority values of $0.1843,0.1766$, and 0.1701 , respectively. Thus, the present approach results in a preference for these microprotocols, which, indeed, are the ones that preferentially target the audio.

\section{Scenario 3: Network Failures}

While in the previous scenario, situations where one QoP parameter was "demonstrably important" with respect to all other QoP and QoS parameters were dealt with, in this scenario, the cases whereby one QoS parameter is now "demonstrably important" with respect to all the other parameters considered in the present model were considered. This situation is not farfetched and can easily arise in real-life situations, particularly when component parts of networks fail or malfunction. Thus, for instance, if a link between two routers goes down, then connections using that link will experience a high degree of segment loss; alternatively, if there is a fault in router hardware, then connections involving that router might, for instance, experience high bit error rates. It must be mentioned, though, that failure or malfunction of network components is not the only possible scenario here: A less dramatic situation, where there is no such failure or malfunction, but where 
TABLE $X$

JUDGEMENT MATRIX DESCRIBING THE RELATIVE IMPORTANCE OF QOS AND QoP PARAMETERS FOR High NeTWORK DElay SCENARIOS

\begin{tabular}{llllllllll}
\hline \hline & BER & SO & SL & DEL & JIT & $V$ & $A$ & $T$ & $D$ \\
\hline BER & EQI & SLI & SLI & DLI & ELI & EQI & EQI & EQI & EQI \\
SO & SMI & EQI & EQI & DLI & SLI & EQI & EQI & EQI & EQI \\
SL & SMI & EQI & EQI & DLI & SLI & EQI & EQI & EQI & EQI \\
DEL & DI & DI & DI & EQI & DI & DI & DI & DI & DI \\
JIT & EI & SMI & SMI & DLI & EQI & EQI & EQI & EQI & EQI \\
$V$ & EQI & EQI & EQI & DLI & EQI & EQI & ELI & WLI & SLI \\
$A$ & EQI & EQI & EQI & DLI & EQI & EI & EQI & SMI & SMI \\
$T$ & EQI & EQI & EQI & DLI & EQI & WMI & SLI & EQI & EQI \\
$D$ & EQI & EQI & EQI & DLI & EQI & SMI & SLI & EQI & EQI \\
\hline \hline
\end{tabular}

TABLE XI

RANKING ORDER OF THE MICROPROTOCOLS AND CORRESPONDING Overall Priority $P_{j}(j=1, \ldots, 9)$ When Delay IS "DEMONSTRABLY IMPORTANT"

\begin{tabular}{cc}
\hline \hline Microprotocol & Overall Score \\
\hline micro1 & 0.1525 \\
micro2 & 0.1383 \\
micro7 & 0.1342 \\
micro4 & 0.1263 \\
micro2 & 0.1198 \\
micro9 & 0.0915 \\
micro6 & 0.0887 \\
micro5 & 0.0833 \\
micro8 & 0.0654 \\
\hline \hline
\end{tabular}

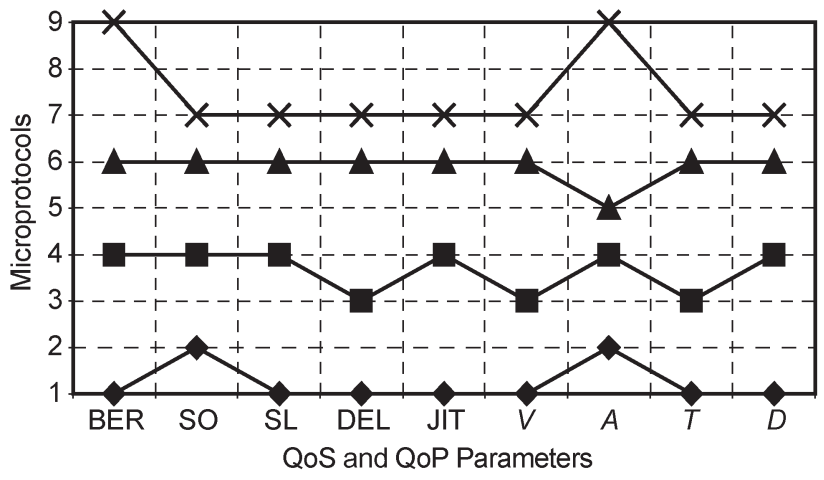

Fig. 4. Resulting DRoPS protocol stack when QoS and QoP parameters are, in turn, "demonstrably important".

connections experience high levels of delay (due to network congestion), is the norm rather than the exception in networks such as the Internet.

Table $\mathrm{X}$ reflects the situation where high levels of delay were detected on the network. The mechanism for evaluating the relative scores $\left(w_{j, i}\right)$ and the priority weights $\left(w_{i}\right)$ is the same as in the previous scenario. The overall priority values when delay is "demonstrably important" are calculated by applying (3), and they are shown in Table XI.

By analyzing the results in Table XI, one can see that the first ranked microprotocol is indeed able to best handle the respective networking scenario. Thus, the "no sequence control" microprotocol (micro1), because of its streamlined functionality, is the protocol that introduces the least amount of delay in the transmission of multimedia in the DRoPS framework.

Fig. 4 shows the resulting protocol stack, which is constructed using the present approach in the DRoPS framework,
TABLE XII

QoP VIDEO CATEGORIES

\begin{tabular}{lcccc}
\hline \hline Video Category & Video & Audio & Text & Dynamism \\
\hline Action movie & $\mathrm{H}$ & $\mathrm{M}$ & $\mathrm{L}$ & $\mathrm{H}$ \\
Chorus & $\mathrm{M}$ & $\mathrm{H}$ & $\mathrm{L}$ & $\mathrm{L}$ \\
Pop music & $\mathrm{H}$ & $\mathrm{H}$ & $\mathrm{H}$ & $\mathrm{M}$ \\
Weather forecast & $\mathrm{H}$ & $\mathrm{H}$ & $\mathrm{H}$ & $\mathrm{L}$ \\
\hline \hline
\end{tabular}

when each of the QoP and QoS parameters becomes, in turn, "demonstrably important" with respect to all the others. Thus, in the case where SL is "demonstrably important," then, as can be seen in Fig. 4, the DRoPS protocol stack is made up of micro1, micro4, micro6, and micro7. While the choice of micro6 is to be expected, as it is the only microprotocol in the DRoPS framework explicitly able to handle losses, the choice of micro4 highlights the importance of flow control for segment losses, which would prevent, for instance, buffer overflows and the resulting loss of data. Otherwise, the choice of micro1 and micro7 reflect the streamlined functionality of the protocol stack, as these microprotocols, by not acting on sequence control and bit errors, respectively, reduce computational overhead.

Similar observations apply in the case when QoP parameters are "demonstrably important." Accordingly, all media component of multimedia presentations are tolerant to bit errors, except audio. Thus, the case when audio is considered "demonstrably important" is the only one in which the resulting protocol stack includes in its configuration micro9, the most suited microprotocol to handle bit errors. The fact that most distributed multimedia applications have real-time constraints, as well as being tolerant to bit errors, is reflected in the choice of the "no-frills" micro7 in all other cases for this type of functionality. The delay-intolerant nature of distributed multimedia applications is also reflected in the choices of microl and micro3 in the suggested protocol stacks when video and text are "demonstrably important." The choice of micro6 for these two scenarios reflects the importance of not losing segments of information, particularly in the case of compressed media, as any loss of information would propagate through subsequent media units, bearing in mind the widespread exploitation of differential characteristics here.

\section{Scenario 4: Content-Dependent Adaptation}

The last scenario considered was initially presented in [1]. In this example, users were presented with a series of four motion pictures expert group (MPEG) video clips (action movie, chorus, pop music, and weather forecast, as detailed in Fig. 1), representing a variety of subject matter, and were asked to rate these clips according to the four QoP criteria listed above, with each QoP criterion being rated on a scale of 1-7. The average results thus obtained for each clip were categorized into "low," "medium," and "high," as depicted in Table XII.

Afterwards, this scale was mapped to the set $\{1,3,9\}$. For instance, if the user judged the importance of the audio $(A)$ component of a clip as high $(=9)$ and the video $(V)$ component as medium $(=3)$, then, in the pairwise comparison matrix, the preference of audio against video will be defined as the ratio of $9 / 3=3$, i.e., weakly more important. The final outcome 
TABLE XIII

Judgement Matrix Describing the Relative Importance of QoS And QoP PARAMEters for the ACtion Movie CliP

\begin{tabular}{llllllllll}
\hline \hline & BER & SO & SL & DEL & JIT & $V$ & $A$ & $T$ & $D$ \\
\hline BER & EQI & SLI & SLI & DLI & ELI & EQI & EQI & EQI & EQI \\
SO & SMI & EQI & EQI & ELI & SLI & EQI & EQI & EQI & EQI \\
SL & SMI & EQI & EQI & ELI & SLI & EQI & EQI & EQI & EQI \\
DEL & DI & EI & EI & EQI & SMI & EQI & EQI & EQI & EQI \\
JIT & EI & SMI & SMI & SLI & EQI & EQI & EQI & EQI & EQI \\
$V$ & EQI & EQI & EQI & EQI & EQI & EQI & WMI & AI & EQI \\
$A$ & EQI & EQI & EQI & EQI & EQI & WLI & EQI & WMI & WLI \\
$T$ & EQI & EQI & EQI & EQI & EQI & ALI & WLI & EQI & ALI \\
$D$ & EQI & EQI & EQI & EQI & EQI & EQI & WMI & AI & EQI \\
\hline \hline
\end{tabular}

TABLE XIV

Results of Four Video Category Examples

\begin{tabular}{lccccccccc}
\hline \hline & & \multicolumn{9}{c}{ Micro-Protocol Ranking Order } & $\mathbf{5}$ & $\mathbf{8}$ & $\mathbf{9}$ \\
\hline Action & $\mathbf{1}$ & $\mathbf{2}$ & $\mathbf{3}$ & $\mathbf{4}$ & $\mathbf{5}$ & $\mathbf{6}$ & $\mathbf{7}$ & micro8 \\
movie & 0.1652 & 0.1374 & 0.1269 & 0.1250 & 0.1166 & 0.0969 & 0.0833 & 0.0809 & 0.0678 \\
\multirow{2}{*}{ Chorus } & micro2 & micro1 & micro4 & micro3 & micro7 & micro9 & micro6 & micro5 & micro8 \\
Pop & 0.1405 & 0.1357 & 0.1345 & 0.124 & 0.116 & 0.1089 & 0.0863 & 0.0855 & 0.0686 \\
music & micro1 & micro3 & micro4 & micro7 & micro2 & micro9 & micro5 & micro6 & micro8 \\
\multirow{2}{*}{ Weather } & 0.1551 & 0.1453 & 0.1343 & 0.1268 & 0.1231 & 0.0939 & 0.0796 & 0.0795 & 0.0624 \\
& micro1 & micro3 & micro7 & micro2 & micro4 & micro9 & micro6 & micro5 & micro8 \\
& 0.1573 & 0.147 & 0.1243 & 0.1236 & 0.1205 & 0.1001 & 0.0826 & 0.0813 & 0.0635 \\
\hline \hline
\end{tabular}

is shown in Table XIII, which details the criteria with respect to criteria matrix for the action movie clip. As can be seen, when comparing this matrix with the one given in Table IV, the only part that has suffered modification is the QoP part. Similar considerations apply to the other clips used in the experiments, and a priori perceptual characterizations of multimedia clips were constructed, according to the perceived importance of the four core QoP components. Of course, during the transmission of the clip, the user, can, if desired, change these a priori characterizations to ones that suit his/her taste.

By applying the same methodology as in the previous scenarios, the priority vectors of these matrices are first obtained, followed afterwards by the overall priority values of each microprotocol. The overall priorities are presented in Table XIV in ranking order, starting with the most suitable microprotocol. Based on these results, the DRoPS protocol stack is constructed by choosing, for each of the four main functionalities provided by the DRoPS framework, the most appropriate microprotocol. Thus, for implementing sequence control, the choice will be made between microprotocols 1 and 2, while, for implementation of checksums, the choice will be made between microprotocols 7, 8, and 9, as detailed in Table I. In the particular case of the pop music clip, for instance, sequence control will be implemented through microl (as it has an overall priority of 0.1551 , which is higher than the priority of 0.1231 associated with micro2, the alternative microprotocol for implementing this functionality).

Similar considerations apply to the choice of the other microprotocols in the construction of the DRoPS communication protocol stack and Fig. 5 depicts the resulting stack after applying the present approach to the four different multimedia video clips. Thus, the present method results in application-specific multimedia communication protocol stacks: In the particular case of the pop music clip, for instance, this stack is made up of micro1, micro3, micro5, and micro7.

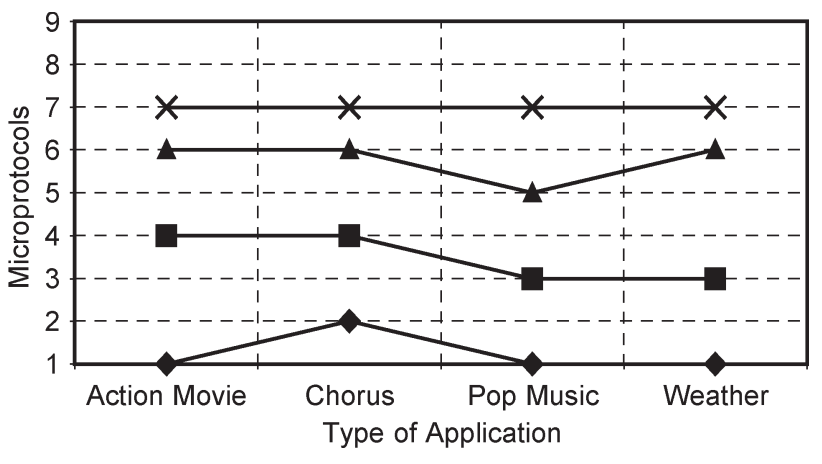

Fig. 5. Content-dependent DRoPS protocol stack.

Fig. 5 also depicts how the constructed protocol stack might vary dynamically according to the multimedia content being visualized: if a user, for instance, is trying to choose between different content channels (movie channel-classical music channel-pop channel-news channel).

\section{CONCLUSION}

A method of obtaining, in the context of a multimedia application, a priority order of low-level QoS parameters, which would ensure that user-level QoP is maintained at an optimum level, has been presented. In the present case, such QoP management would be ensured through dynamic protocol adaptation as a result of changes in user preferences or the operating environment. While the range of multimedia content can be expanded, this does not belittle the implications of this research-in the present approach, based on previously conducted QoP experiments, users do not necessarily need to specify their preferences, with adaptation being done transparently. Moreover, this approach incorporates a human's ability to perceive, analyze, and synthesize information and is more 
comprehensive than preceding attempts, which have usually focused on the viewing enjoyment of multimedia clips, ignoring the infotainment duality of multimedia. Lastly, by employing fuzzy assessment of priorities, inconsistencies and uncertain judgements, inherent in the nature of this problem, were handled.

Naturally, there are several ways to extend this work-these would include integration of this work with lower-level adaptation, such as that characteristic of routing algorithms and network load balancing, as well as the formulation of pairwise relations using linguistic variables, both of which lie at the core of the authors' future endeavors.

\section{REFERENCES}

[1] G. Ghinea and J. P. Thomas, "QoS impact on user perception and understanding of multimedia video clips," in Proc. Association Computing Machinery (ACM) Multimedia, Bristol, U.K., 1998, pp. 49-54.

[2] G. Ghinea, J. P. Thomas, and R. S. Fish, "Quality of perception to quality of service mapping using a dynamically reconfigurable communication system," in Proc. IEEE Global Telecommunications (Globecom), Rio de Janeiro, Brazil, 1999, pp. 2061-2065.

[3] R. T. Apteker, J. A. Fisher, V. S. Kisimov, and H. Neishlos, "Video acceptability and frame rate," IEEE Multimed. Mag., vol. 2, no. 3, pp. 32-40, Fall 1995.

[4] D. Wijesekera and J. Srivastava, "Quality of service (QoS) metrics for continuous media," Multimed. Tools Appl., vol. 3, no. 1, pp. 127-166, 1996.

[5] R. Steinmetz, "Human perception of jitter and media synchronisation," IEEE J. Sel. Areas Commun., vol. 14, no. 1, pp. 61-72, Jan. 1996.

[6] A. Watson and M. A. Sasse, "Measuring perceived quality of speech and video in multimedia conferencing applications," in Proc. Association Computing Machinery (ACM) Multimedia, Bristol, U.K., 1998, pp. 55-60.

[7] R. De Silva, B. Landfeldt, S. Ardon, A. Seneviratne, and C. Diot, "Managing application level quality of service through TOMTEN," Comput. Netw., vol. 31, no. 7, pp. 727-739, Nov.-Dec. 1999.

[8] D. Reininger, D. Raychaudhuri, and M. Ott, "A dynamic quality of service framework for video in broadband networks," IEEE Networks Mag., vol. 12 , no. 6 , pp. 22-34, 1998.

[9] A. Seneviratne, M. Fry, V. Witana, V. Saparamadu, A. Richards, and E. Horlait, "Quality of service management for distributed multimedia applications," in Proc. IEEE Communication Society Phoenix Conf. Computers and Communications, Phoenix, AZ, 1994, p. 434.

[10] D. J. Simons, "Attentional capture and inattentional blindness," Trends Cogn. Sci., vol. 4, no. 4, pp. 147-155, 2000.

[11] K. Nahrstedt, "End-to-end QoS guarantees in networked multimedia systems," ACM Comput. Surv. J., vol. 27, no. 4, pp. 613-616, 1995.

[12] R. S. Fish and R. J. Loader, "A kernel based adaptable protocol architecture," in Proc. 24th Euromicro Conf., Västerås, Sweden, 1998, pp. 1029-1036.

[13] T. L. Saaty, The Analytic Hierarchy Process. New York: McGraw-Hill, 1980.

[14] - , "A scaling method for priorities in hierarchical structures," J. Math. Psychol., vol. 15, no. 3, pp. 234-281, 1977.

[15] J. J. Buckley, "Fuzzy hierarchical analysis," Fuzzy Sets Syst., vol. 17, no. 3, pp. 233-247, 1985.

[16] R. Islam, M. P. Bismal, and S. S. Alam, "Theory and methodology: Preference programming and inconsistent interval judgements," Eur. $J$. Oper. Res., vol. 97, no. 1, pp. 53-62, 1997.

[17] A. Arbel, "Theory and methodology: Approximate articulation of preference and priority derivation," Eur. J. Oper. Res., vol. 43, no. 3, pp. 317-326, 1989.

[18] G. A. Miller, "The magical number seven plus or minus two: Some limits on our capacity for processing information," Psychol. Rev., vol. 63, no. 2, pp. 81-97, 1956.
[19] E. Triantaphyllou and C.-T. Lin, "Development and evaluation of five fuzzy multiattribute decision-making methods," Int. J. Approx. Reason., vol. 14, no. 4, pp. 281-310, 1996.

[20] N. Bryson, "A goal programming method for generating priority vectors," J. Oper. Res. Soc., vol. 46, no. 5, pp. 641-648, 1995.

[21] L. Mikhailov and M. G. Singh, "Fuzzy assessment of priorities with application to the competitive bidding," J. Decis. Syst., vol. 8, no. 1, pp. 11-28, 1999.

[22] $\longrightarrow$, "Comparison analysis of methods for deriving priorities in the analytic hierarchy process," in Proc. IEEE Int. Conf. Systems, Man and Cybernetics, Tokyo, Japan, 1999, pp. 1037-1042.

[23] G. Ghinea and G. D. Magoulas, "Perceptual considerations for quality of service management: An integrated architecture," in Proc. 8th Int. User Modeling Conf., Sonthofen, Germany, 2001, vol. 2109, pp. 234-236.

[24] — "Quality of service for perceptual considerations: An integrated perspective," in Proc. IEEE Int. Conf. Multimedia and Expo (ICME), Tokyo, Japan, 2001, pp. 752-755.

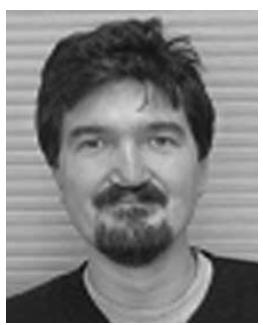

Gheorghita Ghinea (M'02) received the B.Sc. and B.Sc.(Hons.) degrees in computer science and mathematics in 1993 and 1994, respectively, and the M.Sc. degree in computer science in 1996, from the University of the Witwatersrand, Johannesburg, South Africa. He received the Ph.D. degree in computer science from the University of Reading, Reading, U.K., in 2000.

$\mathrm{He}$ is a Lecturer at the Department of Information Systems and Computing, Brunel University, Uxbridge, Middlesex, U.K. His research interests span perpetual aspects of multimedia, quality of service, and multimedia resource allocation, as well as computer networking and security issues.

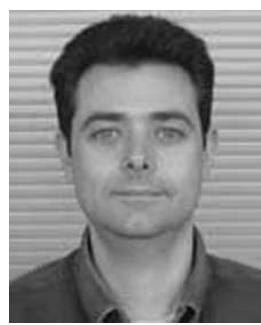

George D. Magoulas (M'02) received the B.Eng./ M.Eng. and Ph.D. degrees in electrical and computer engineering from the University of Patras, Patras, Greece, in 1990 and 1998, respectively.

He held Lecturer and Senior Lecturer posts at the Department of Information Systems and Computing, Brunel University, Uxbridge, Middlesex, U.K. He is currently a Reader in computer science at the School of Computer Science and Information Systems, Birkbeck College, University of London, London, U.K. His research is focused on learning and evolution algorithms with applications to intelligent adaptive systems. His work is currently funded by the E.P.S.R.C. and the A.H.R.B, U.K.

Dr. Magoulas is a member of User Modeling Inc., the Technical Chamber of Greece, and the Hellenic Artificial Intelligence Society.

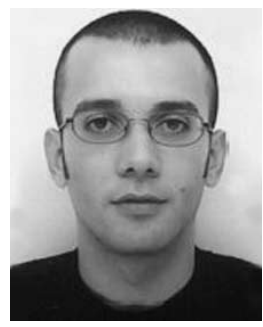

Christos Siamitros received the B.Sc. degree in business mathematics with applicable mathematics (A-Class Hons.) from Manchester Metropolitan University, Manchester, U.K., in 1999, the M.Sc. degree in computation from the University of Manchester Institute of Science and Technology, Manchester, U.K., in 2000, and the Ph.D. degree from the Department of Mathematical Sciences, Brunel University, Uxbridge, Middlesex, U.K., in 2004.

His research interests are in the areas of modelmulticriteria decision making.

ing for discrete optimization problems and in fuzzy 